\title{
BMJ Open Optimal duration of prone positioning in patients with acute respiratory distress syndrome: a protocol for a systematic review and meta-regression analysis
}

\author{
Tetsuro Kamo, ${ }^{1,2}$ Yoshitaka Aoki, ${ }^{3}$ Tatsuma Fukuda, ${ }^{4,5}$ Kiyoyasu Kurahashi, ${ }^{6}$ \\ Hideto Yasuda, ${ }^{7,8}$ Masamitsu Sanui, ${ }^{9}$ Eishu Nango, ${ }^{10}$ Takayuki Abe, ${ }^{8,11}$ \\ Alan Kawarai Lefor, ${ }^{12}$ Satoru Hashimoto ${ }^{13}$
}

To cite: Kamo T, Aoki Y, Fukuda T, et al. Optimal duration of prone positioning in patients with acute respiratory distress syndrome: a protocol for a systematic review and metaregression analysis. BMJ Open 2018;8:e021408. doi:10.1136/ bmjopen-2017-021408

- Prepublication history for this paper is available online. To view these files, please visit the journal online (http://dx.doi. org/10.1136/bmjopen-2017021408).

Received 1 January 2018 Revised 18 July 2018 Accepted 27 July 2018
Check for updates

(c) Author(s) (or their employer(s)) 2018. Re-use permitted under CC BY-NC. No commercial re-use. See rights and permissions. Published by BMJ.

For numbered affiliations see end of article.

Correspondence to Professor Masamitsu Sanui; msanui@mac.com

\section{ABSTRACT}

Introduction Several systematic reviews and metaanalyses have demonstrated that prolonged ( 216 hours) prone positioning can reduce the mortality associated with acute respiratory distress syndrome (ARDS). However, the effectiveness and optimal duration of prone positioning was not fully evaluated. To fill these gaps, we will first investigate the effectiveness of prone positioning compared with the conventional management of patients with ARDS, regarding outcomes using the Grading of Recommendations Assessment, Development, and Evaluation (GRADE) system. Second, if statistical heterogeneity in effectiveness with regard to shortterm mortality (intensive care unit death or $\leq 30$-day mortality) is shown, we will conduct a meta-regression analysis to explore the association between duration and effectiveness, and determine the optimal duration of prone positioning.

Method and analysis Relevant studies are collected using PubMed/MEDLINE, Embase, Cochrane Central Register of Controlled Trials and the WHO International Clinical Trials Platform Search Portal. Randomised controlled trials comparing prone and supine positioning in adults with ARDS will be included in the meta-analysis. Two independent investigators will screen trials obtained by search eligibility and extract data from selected studies to standardised data recording forms. For each selected trial, the risk of bias and quality of evidence will be evaluated using the GRADE system. Meta-regression analyses will be performed to identify the most important factors associated with short-term mortality, and subgroup analysis will be used to analyse the following: duration of mechanical ventilation in the prone position per day, patient severity, tidal volume and cause of ARDS. If heterogeneity or inconsistency among the studies is detected, subgroup analysis will be conducted on factors that may cause heterogeneity.

Ethics and dissemination This study requires no ethical approval. The results obtained from this systematic review and meta-analysis will be disseminated through international conference presentations and publication in a peer-reviewed journal.
Strengths and limitations of this study

- One strength of this study is that it is a systematic review with meta-regression analysis comparing prone positioning to other positions for patients with acute respiratory distress syndrome undergoing mechanical ventilation.

- The Grading of Recommendations, Assessment, Development and Evaluation system will be used to assess the strength of the evidence base and allow clinicians to judge the quality of available evidence.

- We plan sensitivity analyses and meta-regression to examine the relationship between the duration of prone positioning and its efficacy.

- Non-English articles will not be included in our study due to language difficulties which may result in publication bias.

- A possible weakness may be the quantity and quality of the trials we identify.

PROSPERO registration number CRD42017078340.

\section{INTRODUCTION}

More than 200000 patients are diagnosed with acute respiratory distress syndrome (ARDS) each year accounting for 3.6 million hospital-days of annual admissions in the USA. ${ }^{1}$ The prevalence of ARDS is approximately $10 \%$ of all intensive care unit (ICU) admissions $^{2}$ and treating ARDS comprises $5 \%$ of all hospital ventilator-days, resulting in enormous medical expenses, up to US $\$ 115000 /$ hospital stay. ${ }^{34}$ Despite advances in the ventilator management of patients with ARDS, ${ }^{5}$ mortality rates of patients with moderate to severe ARDS still remain as high as $30 \%-40 \% .^{16}$

Prone positioning has been used to manage patients with ARDS since a study 
in 1976 reported improved oxygenation from prone positioning. ${ }^{7}$ Physiological studies showed improved oxygenation after prone positioning in a majority of patients with ARDS, ${ }^{8}{ }^{9}$ but randomised controlled trials (RCTs) failed to show a significant reduction in mortality with prone positioning. ${ }^{10-12}$ Of recent RCTs examining the efficacy of prone positioning for patients with ARDS, ${ }^{13-15}$ the PROSEVA study ${ }^{15}$ published in 2013, an RCT treating patients with severe ARDS with prolonged ( $\geq 16$ hours) prone positioning, showed an improvement in mortality rates. Several systematic reviews and meta-analyses of studies including these RCTs indicate that prone positioning may reduce the mortality rates in patients with ARDS, especially those with severe hypoxaemia. ${ }^{16-20}$ Although the duration of prone positioning appears to affect patient outcomes, the relationship between the duration and its efficacy, and the shortest duration needed to improve outcomes are unknown.

Previous systematic reviews and meta-analyses ${ }^{17-21}$ have shown that prolonged prone positioning $(\geq 10,12$ or 16 hours/day) may be effective in patients with ARDS. However, these studies did not conduct meta-regression analyses to investigate the potential heterogeneity of the results or meticulous subgroup analyses using a strict systematic approach such as the Grading of Recommendations Assessment, Development, and Evaluation (GRADE) system. ${ }^{22}$ We will conduct meta-regression analyses to examine associations between effect sizes and variables that may influence short-term mortality, such as patient characteristics, duration of prone positioning, tidal volume and the use of neuromuscular blocking agents.

\section{OBJECTIVE}

The objective of this systematic review and meta regression analysis is to investigate the duration of prone positioning needed to improve outcomes using sensitivity analyses and meta-regression.

\section{METHODS AND ANALYSES}

This systematic review will be conducted according to the Cochrane Handbook for Systematic Review of Interventions, the Preferred Reporting Items for Systematic Reviews and Meta-Analyses (PRISMA) statement, and the GRADE system. ${ }^{22-24}$ The logistics and reporting of this protocol will be in compliance with PRISMA-P.Meta-regression is thought to be meaningful only with more than 10 studies included in the analysis. ${ }^{23}$

\section{Patient and public involvement}

No patients were involved in the design of the study. We will submit our results to a peer-reviewed journal for publication to enable dissemination.

\section{STUDY ELIGIBILITY}

\section{Type of studies}

Published and unpublished RCTs and randomised crossover trials (the first-period only) between January 1980 and September 2017 were included, restricted to the English language. Quasi-experimental studies and cluster randomisations were excluded. We will only include RCTs with supine positioning or semirecumbent position (which could include lateral positioning as part of routine pressure care) for ARDS and acute lung injury. We will exclude studies examining rotational bed therapies.

\section{Type of participants}

This study will include adults with ARDS or acute lung injury from any cause, as defined by the North-American-European Consensus Conference on $\mathrm{ARDS}^{25}$ and the Berlin definition, ${ }^{7}$ aged 16 years or older, undergoing mechanical ventilation. Cointerventions in addition to prone positioning will be permitted. We excluded studies of neonates or paediatric patients (ie, younger than 16 years), and also excluded duplicated studies or data, studies using specific treatment options including high-frequency oscillatory ventilation, ${ }^{26}{ }^{27}$ inhaled nitric oxide, ${ }^{28}$ extracorporeal membrane oxygenation and studies without sufficient data regarding outcomes. ${ }^{29}$

\section{Type of interventions and comparators}

The intervention of interest is the initiation of prone positioning, regardless of the duration. The comparator group will contain all positions other than prone positioning during mechanical ventilation.

\section{Type of outcomes}

The following outcome measures will be evaluated: the primary outcomes are short-time mortality (ICU deaths or $\leq 30$-day mortality) and endotracheal tube malfunction (unplanned extubation, dislocation or obstruction of the endotracheal tube). Secondary outcomes are the number of ventilator-free days up to 28 days, the incidence of ventilator-associated pneumonia and decubitus ulcers.

\section{INFORMATION SOURCES}

Two investigators (TK, YA) will search for the eligible trials from the following databases:

1. The Cochrane Central Register of Controlled Trials.

2. Ovid/MEDLINE.

3. Embase.

4. The WHO International Clinical Trials Platform Search Portal.

We will also check the reference lists in the relevant sections of international guidelines. ${ }^{30}$ We will search the reference lists of relevant studies and studies cited in studies using Web of Science. ${ }^{31}$

\section{SEARCH STRATEGY}

Investigators will search the keywords 'prone position' AND 'ARDS', 'adult respiratory distress syndrome', 'ALI' 
or 'acute lung injury'. We will also perform a MeSH term search using the following terms: 'respiratory distress syndrome, adult', or 'acute lung injury' or 'lung injury' AND 'Prone position'. Searches will be performed from 18 July to 31 July 2018 . The detailed strategy and details of the dates performed are shown in box 1 .

\section{Study records and data management}

Literature selected from each database will be extracted into Microsoft Excel files and duplicates will be removed by sorting the data alphabetically according to author. The results of all processes (first and second screenings) are entered into the same data file. All full-text files will be managed with Papers bibliographic software. For studies lacking information, we will directly contact the corresponding author of each study to request the information.

Meta-analysis and meta-regression analysis will be conducted with Review Manager (RevMan) software V.5.3.5 $5^{32}$ and the graphical user interface for R (The R Foundation for Statistical Computing, Vienna, Austria). ${ }^{33}$ All data will be managed by the primary investigator (TK).

\section{Selection process}

Two investigators (TK, YA) will screen titles and abstracts as the first screening process, and the full text as secondary screening for relevant studies and will then independently extract data from included studies to standardised data forms. HY will supervise the process of systematic review. TA will supervise the process of analysis as a biostatistician. MS and SH are consultants on clinically relevant issues.

\section{Data collection process}

After the second screening, data will be extracted from each study by two investigators (TK, YA) using two tools: the Cochrane Data Collection Form (RCTs only) ${ }^{34}$ and Review Manager (RevMan) software V.5.3.5. ${ }^{32}$

\section{Risk of bias in individual studies}

Investigators will assess the risk of bias in each selected study based on a modified version of the Cochrane riskof-bias instrument. ${ }^{35}$ The risk of bias will be evaluated for random sequence generation (selection bias), allocation concealment (selection bias), blinding of participants and personnel (performance bias), blinding of outcome assessment (detection bias), incomplete outcome data (attrition bias), selective reporting (reporting bias) and other relevant potential bias (cross over). Two investigators (TK, YA) will independently conduct study selection, data extraction and risk of bias assessment. Two investigators will resolve disagreements between the two investigators through discussion, with a third reviewer available for adjudication if needed (HY).

\section{Data analysis}

Data synthesis

Statistical analyses will be performed using Comprehensive Meta-Analysis using Review Manager (RevMan) 5.5.5. ${ }^{32}$ We will use a fixed-effect meta-analysis except

\section{Box 1}

(A) Ovid/MEDLINE

1. exp Lung Injury/

2. Acute respiratory distresss.mp.

3. Adult Respiratory distresss.mp.

4. ARDS.mp.

5. acute lung injury.mp.

6. acute lung injuries.mp.

7. shock lung.mp.

8. \#1 OR \#2 OR \#3 OR \#4 OR \#5 OR \#6 OR \#7

9. exp Prone Position/

10. prone* position*.mp.

11. $\# 9$ OR \#10

12. \#8 AND \#11

13. randomized controlled trial.pt.

14. controlled clinical trial.pt.

15. randomi?ed.ab.

16. placebo.ab.

17. clinical trials as topic.sh.

18. randomly.ab.

19. trial.ti.

20. drug therapy.sh.

21. groups.ab.

22. or $/ 13-22$

23. exp animals/ not humans.sh.

24. 22 not 23 \#25 and/12,24

(B) Embase

1. 'adult respiratory distress syndrome'/exp

2. 'acute lung injury'/exp

3. 'lung injury'/exp

4. 'acute respiratory distress' $O R$ 'adult respiratory distress' $O R$ ards $\mathrm{OR}$ 'acute lung injury' $\mathrm{OR}$ 'acute lung injuries' $\mathrm{OR}$ 'shock lung'

5. $\mathrm{OR} / \# 1-\# 4$

6. 'prone position'/exp

7. prone* AND position*

8. $0 \mathrm{R} / \# 6-\# 7$

9. \#5 AND \#8

10. 'controlled clinical trial'/exp

11. 'randomized controlled trial'/exp

12. randomized:ab,ti

13. randomly:ab,ti

14. trial:ab,ti

15. placebo:ab,ti

16. groups:ab,ti

17. $\mathrm{OR} / \# 10-\# 16$

18. 'animal'/exp

19. 'invertebrate'/exp

20. 'animal experiment'

21. 'animal model'

22. 'animal tissue'

23. 'animal cell'

24. nonhuman

25. $0 \mathrm{R} / \# 18-\# 24$

26. human

27. 'human cell'

28. $\mathrm{OR} / \# 24-\# 25$

29. \#25 AND \#28

30. \#25 NOT \#29

31. \#17 NOT \#30

32. \#9 AND \#31 


\section{Box 1 Continued}

(C) Cochrane Central Register of Controlled Trials

1. MeSH descriptor: [Respiratory Distress Syndrome, Adult] explode all trees

2. MeSH descriptor: [Acute Lung Injury] explode all trees

3. MeSH descriptor: [Lung Injury] explode all trees

4. Acute respiratory distress:ti,ab,kw or Adult respiratory distress:ti,ab,kw or ARDS:ti,ab,kw or acute lung injury:ti,ab,kw or acute lung injuries:ti,ab,kw or shock lung:ti,ab,kw

5. \#1 or \#2 or \#3 or \#4

6. MeSH descriptor: [Prone Position] explode all trees

7. prone ${ }^{*}$ position ${ }^{\star}: t i, a b, k w$

8. \#6 or \#7

9. \#5 and \#8

10. \#9 and in Trials

(D) The World Health Organization International Clinical Trials Platform Search Portal

1. Respiratory Distress Syndrome

2. Acute Lung Injury

3. Lung Injury

4. \#1 OR \#2 OR \#3

5. prone

when we identified statistical heterogeneity, and then used a random-effects model.

\section{Continuous data}

Continuous data will be presented as a mean difference with 95\% CIs. Pooled effect estimates will be stated with 95\% CIs quantitatively and illustrated in a forest plot along with tables where necessary. ${ }^{36}$ The data reported as medians will be converted to means and the range/ 4 will be converted to SD if possible. ${ }^{37}$

\section{Categorical data}

For categorical data, results will be expressed as a pooled relative risk with $95 \%$ CI.

\section{Assessment of heterogeneity}

Inconsistency (heterogeneity) among included studies will be assessed by examination of forest plots and the $\mathrm{I}^{2}$ statistics. ${ }^{38}$ We will be considered statistical heterogeneity to be low for $\mathrm{I}^{2} \leq 40 \%$, moderate for $\mathrm{I}^{2}=30 \%-60 \%$, substantial for $\mathrm{I}^{2}=50 \%-90 \%$ and considerable for $\mathrm{I}^{2}=75 \%-100 \%$. Cochran's $Q$ statistic will be used for quantifying heterogeneity. The statistical analysis for publication bias will be planned for outcomes with at least 10 included studies. ${ }^{23}$ If there are any kinds of heterogeneity, they will be investigated through sensitivity analyses and meta-regression to explore the potential sources of heterogeneity.

\section{Subgroup analysis}

If heterogeneity or inconsistency among the studies is detected, subgroup analyses will be conducted on the main factors that may cause heterogeneity.

We plan to undertake the following subgroup analyses.

- Duration of ventilation in the prone position per day ( $<8$ hours/day vs $\geq 8$ hours/day).
- Outcomes according to severity (using an index of oxygenation; arterial oxygen tension/fractional inspired oxygen ratio $(\mathrm{P} / \mathrm{F}$ ratio $)(<150 \mathrm{~mm} \mathrm{Hg}$ vs $\geq 150 \mathrm{~mm} \mathrm{Hg}$ ), severity of illness score; Simplified Acute Physiology Score II (SAPS II) $(<50$ vs $\geq 50)$ ).

- Tidal volume $(<8 \mathrm{~mL} / \mathrm{kg}$ of ideal body weight vs $\geq 8 \mathrm{~mL} / \mathrm{kg}$ of ideal body weight).

- Cause of ARDS (pulmonary or extrapulmonary).

We plan to explore differences in outcomes in these subgroups if the number of collected studies are sufficient.

\section{Sensitivity analysis}

We will perform sensitivity analysis depending on study characteristics identified during the review process using fixed-effect model analysis. We will exclude studies with one or more 'low' or 'very low' from the sensitivity analysis. The remaining studies will be used for sensitivity analysis.

\section{Meta-regression}

If there is any statistically significant heterogeneity, or if considerable methodological heterogeneity is noted, investigators will explore the relationship between the duration of prone positioning and the short-term mortality by using random-effects meta-regression. We will perform meta-regression analysis by using the following factors as covariates.

Intervention characteristics:

- Duration of prone positioning (hours).

- Tidal volume $(\leq 8 \mathrm{~mL} / \mathrm{kg}$ of ideal body weight or $>8 \mathrm{~mL} / \mathrm{kg}$ of ideal body weight).

- Using neuromuscular blocking agents or none.

Participant characteristics:

- Mean age.

- SAPS II score.

- Severity of hypoxaemia; $\mathrm{P} / \mathrm{F}$ ratio.

If studies are insufficient to justify meta-regression techniques, we will conduct meta-regression analysis by limiting the covariates.

\section{Assessment of reporting bias}

A funnel plot will be used to investigate the possibility of publication bias if $>10$ studies are available (RevMan). ${ }^{39}$ Egger's test will be performed on each study group to evaluate asymmetry in funnel plots. ${ }^{40}$

\section{Assessment of evidence in cumulative evidence}

We will assess and rate the quality of evidence for each outcome across studies using four levels (high, moderate, low or very low) according to the GRADE criteria. ${ }^{41}$

The quality of evidence will be decreased by any one of the following limitations: risk of bias, imprecision, inconsistency, indirectness and publication bias. Two investigators (TK, YA) will independently conduct study selection, data extraction and risk of bias assessment. Investigators will resolve disagreements between the two investigators through discussion, with a third reviewer available for adjudication if needed (HY). 
Author affiliations

${ }^{1}$ Department of Pulmonary Medicine, Intensive Care Medicine, Keio University School of Medicine, Tochigi, Japan

${ }^{2}$ Department of Pulmonary Medicine, Keio University School of Medicine, Tokyo, Japan

${ }^{3}$ Department of Anesthesiology and Intensive Care Medicine, Shizuoka General Hospital, Shizuoka, Japan

${ }^{4}$ Department of Emergency and Critical Care Medicine, Graduate School of

Medicine, The University of Tokyo, Tokyo, Japan

${ }^{5}$ Department of Emergency Medicine, Beth Israel Deaconess Medical Center, Harvard Medical School, Boston, Massachusetts, USA

${ }^{6}$ Department of Anesthesiology and Intensive Care Medicine, School of Medicine, International University of Health and Welfare, Chiba, Japan

${ }^{7}$ Department of Intensive Care Medicine, Kameda Medical Center, Chiba, Japan ${ }^{8}$ Department of Preventive Medicine and Public Health, Keio University School of Medicine, Tokyo, Japan

${ }^{9}$ Department of Anesthesiology and Critical Care Medicine, Jichi Medical University Saitama Medical Center, Saitama, Japan

${ }^{10}$ Department of General Medicine, Tokyo-Kita Medical Center, Tokyo, Japan

${ }^{11}$ Biostatistics Unit at Clinical and Translational Research Center, Keio University Hospital, Tokyo, Japan

${ }^{12}$ Department of Surgery, Jichi Medical University, Tochigi, Japan

${ }^{13}$ Department of Anesthesiology and Intensive Care Medicine, Kyoto Prefectural University of Medicine, Kyoto, Japan

Contributors TF and MS contributed to the conception of the study. TK, YA, TF, $\mathrm{KK}$ and $\mathrm{HY}$ designed the model and the framework of the systematic review and will analyse the data under supervisions of MS, EN and TA. TK wrote the protocol in consultation with YA, MS, AKL and SH. All authors were involved in the critical revision, for the intellectual content, and read and approved the final manuscript

Funding This research received no specific grant from any funding agency in the public, commercial or not-for-profit sectors.

Competing interests None declared.

Patient consent Not required.

Provenance and peer review Not commissioned; externally peer reviewed.

Open access This is an open access article distributed in accordance with the Creative Commons Attribution Non Commercial (CC BY-NC 4.0) license, which permits others to distribute, remix, adapt, build upon this work non-commercially, and license their derivative works on different terms, provided the original work is properly cited, appropriate credit is given, any changes made indicated, and the use is non-commercial. See: http://creativecommons.org/licenses/by-nc/4.0/.

\section{REFERENCES}

1. Rubenfeld GD, Caldwell E, Peabody E, et al. Incidence and outcomes of acute lung injury. N Engl J Med 2005;353:1685-93.

2. Bellani G, Laffey JG, Pham T, et al. Epidemiology, patterns of care, and mortality for patients with acute respiratory distress syndrome in intensive care units in 50 countries. JAMA 2016;315:788-800.

3. Cheung AM, Tansey CM, Tomlinson G, et al. Two-year outcomes, health care use, and costs of survivors of acute respiratory distress syndrome. Am J Respir Crit Care Med 2006;174:538-44.

4. Esteban A, Ferguson ND, Meade MO, et al. Evolution of mechanical ventilation in response to clinical research. Am J Respir Crit Care Med 2008;177:170-7.

5. Cochi SE, Kempker JA, Annangi S, et al. Mortality trends of acute respiratory distress syndrome in the United States from 1999 to 2013. Ann Am Thorac Soc 2016;13:1742-51.

6. Piehl MA, Brown RS. Use of extreme position changes in acute respiratory failure. Crit Care Med 1976;4:13-14.

7. Ranieri VM, Rubenfeld GD, Thompson BT, et al. Acute respiratory distress syndrome: the Berlin Definition. JAMA 2012;307:2526-33.

8. Albert RK, Leasa D, Sanderson M, et al. The prone position improves arterial oxygenation and reduces shunt in oleic-acid-induced acute lung injury. Am Rev Respir Dis 1987;135:628-33.

9. Chatte G, Sab JM, Dubois JM, et al. Prone position in mechanically ventilated patients with severe acute respiratory failure. Am J Respir Crit Care Med 1997;155:473-8.

10. Guerin C, Gaillard S, Lemasson S, et al. Effects of systematic prone positioning in hypoxemic acute respiratory failure: a randomized controlled trial. JAMA 2004;292:2379-87.
11. Voggenreiter G, Aufmkolk M, Stiletto RJ, et al. Prone positioning improves oxygenation in post-traumatic lung injury--a prospective randomized trial. J Trauma 2005;59:333-43.

12. Gattinoni L, Tognoni G, Pesenti A, et al. Effect of prone positioning on the survival of patients with acute respiratory failure. $N$ Engl J Med 2001;345:568-73.

13. Taccone $P$, Pesenti $A$, Latini $R$, et al. Prone positioning in patients with moderate and severe acute respiratory distress syndrome: a randomized controlled trial. JAMA 2009;302:1977-84.

14. Chan MC, Hsu JY, Liu HH, et al. Effects of prone position on inflammatory markers in patients with ARDS due to communityacquired pneumonia. J Formos Med Assoc 2007;106:708-16.

15. Guérin C, Reignier J, Richard JC, et al. Prone positioning in severe acute respiratory distress syndrome. $N$ Engl J Med 2013;368:2159-68.

16. Sud S, Friedrich JO, Taccone P, et al. Prone ventilation reduces mortality in patients with acute respiratory failure and severe hypoxemia: systematic review and meta-analysis. Intensive Care Med 2010;36:585-99.

17. Lee JM, Bae W, Lee YJ, et al. The efficacy and safety of prone positional ventilation in acute respiratory distress syndrome: updated study-level meta-analysis of 11 randomized controlled trials. Crit Care Med 2014:42:1252-62.

18. Hu SL, He HL, Pan C, et al. The effect of prone positioning on mortality in patients with acute respiratory distress syndrome: a meta-analysis of randomized controlled trials. Crit Care 2014;18:109.

19. Bloomfield R, Noble DW, Sudlow A. Prone position for acute respiratory failure in adults. Cochrane Database Syst Rev 2015:CD008095

20. Sud S, Friedrich JO, Adhikari NK, et al. Effect of prone positioning during mechanical ventilation on mortality among patients with acute respiratory distress syndrome: a systematic review and metaanalysis. CMAJ 2014;186:E381-E390.

21. Beitler JR, Shaefi S, Montesi SB, et al. Prone positioning reduces mortality from acute respiratory distress syndrome in the low tida volume era: a meta-analysis. Intensive Care Med 2014;40:332-41.

22. Guyatt GH, Oxman AD, Vist GE, et al. GRADE: an emerging consensus on rating quality of evidence and strength of recommendations. BMJ 2008;336:924-6.

23. Higgins JPT, Green S. Cochrane Handbook for Systematic Reviews of Interventions. Version 5.1.0. Oxford, England: cochrane collaboration. 2011 handbook.cochrane.org

24. Moher D, Liberati A, Tetzlaff J, et al. Preferred reporting items for systematic reviews and meta-analyses: the PRISMA statement. BMJ 2009;339:b2535

25. Bernard GR, Artigas A, Brigham KL, et al. The American-European Consensus Conference on ARDS. Definitions, mechanisms, relevant outcomes, and clinical trial coordination. Am J Respir Crit Care Med 1994;149:818-24.

26. Demory D, Michelet P, Arnal JM, et al. High-frequency oscillatory ventilation following prone positioning prevents a further impairment in oxygenation. Crit Care Med 2007;35:106-11.

27. Papazian L, Gainnier M, Marin V, et al. Comparison of prone positioning and high-frequency oscillatory ventilation in patients with acute respiratory distress syndrome. Crit Care Med 2005;33:2162-71.

28. Borelli M, Lampati L, Vascotto E, et al. Hemodynamic and gas exchange response to inhaled nitric oxide and prone positioning in acute respiratory distress syndrome patients. Crit Care Med 2000;28:2707-12.

29. Fernandez R, Trenchs X, Klamburg J, et al. Prone positioning in acute respiratory distress syndrome: a multicenter randomized clinical trial. Intensive Care Med 2008;34:1487-91.

30. Fan E, Del Sorbo L, Goligher EC, et al. An official American Thoracic Society/European Society of Intensive Care Medicine/Society of Critical Care Medicine Clinical Practice Guideline: mechanical ventilation in adult patients with acute respiratory distress syndrome. Am J Respir Crit Care Med 2017;195:1253-63.

31. Web of Science. Please sign in to access web of science. http:// www.webofknowledge.com (accessed 7 Sep 2017).

32. RevMan 5 download and installion. Secondary RevMan 5 download and installion. http://tech.cochrane.org/revman/download (accessed 7 Sep 2017).

33. Ihaka R, Gentleman R. R: a language for data analysis and graphics. J Comput Graph Stat 1996;5:299-314.

34. Cochrane Training. Cochrane handbook for systematic reviews of interventions. http://training.cochrane.org/handbook (accessed 7 Sep 2017).

35. Higgins JP, Altman DG, Gøtzsche PC, et al. The Cochrane Collaboration's tool for assessing risk of bias in randomised trials. BMJ 2011;343:d5928. 
36. DerSimonian R, Laird N. Meta-analysis in clinical trials revisited. Contemp Clin Trials 2015;45:139-45.

37. Hozo SP, Djulbegovic B, Hozo I. Estimating the mean and variance from the median, range, and the size of a sample. BMC Med Res Methodol 2005;5:5-13.

38. Higgins JP, Thompson SG, Deeks JJ, et al. Measuring inconsistency in meta-analyses. BMJ 2003;327:557-60.
39. Sterne JA, Sutton AJ, loannidis JP, et al. Recommendations for examining and interpreting funnel plot asymmetry in meta-analyses of randomised controlled trials. BMJ 2011;343:d4002.

40. Egger M, Davey Smith G, Schneider M, et al. Bias in meta-analysis detected by a simple, graphical test. BMJ 1997;315:629-34.

41. Balshem $\mathrm{H}$, Helfand $\mathrm{M}$, Schünemann $\mathrm{HJ}$, et al. GRADE guidelines: 3. Rating the quality of evidence. J Clin Epidemiol 2011;64:401-6. 